\title{
EQUATIONS IN THE THEORY OF MONADIC ALGEBRAS
}

\author{
TH. LUCAS ${ }^{1}$
}

\begin{abstract}
Identities in the theory of monadic algebras are equivalent to simpler "standard" ones. This property is used to prove some well-known results as well as to determine the minimum number of variables needed in an identity characterizing an equational class. Identities are also shown to be preserved under certain types of extension.
\end{abstract}

In this paper we prove that the equations of the theory of monadic algebras can be "reduced" to simpler "standard" ones. From this reduction, some properties already proved in [2] easily follow (see Theorem 3.3). We also obtain new results, mainly

(1) The determination of the minimum number of variables needed in an equation characterizing a given equational class. (See Theorem 3.4; this question is raised in [2].)

(2) A positive answer for the case $\alpha=1$ to the following two questions: Does every equation holding in a $C A_{\alpha}$ also hold in its canonical embedding algebra? Does it also hold in its completion algebra? (See Corollary 3.6; these questions were raised by Monk at a seminar in algebraic logic at the University of Colorado.)

I wish to thank Donald Monk for much valuable advice and Dan Demaree for useful conversations. I am also indebted to the referee whose suggestions have helped to improve a first version of this paper.

1. Preliminaries. A monadic algebra $\mathfrak{A}$ is considered as a structure $\langle A,+, \cdot,-, C\rangle$ where $\langle A,+, \cdot,-\rangle$ is a boolean algebra and $C$ is a quantifier on $\langle A,+, \cdot,-\rangle . C A_{1}$ is the class of all monadic algebras.

The first-order language $L_{1}$ of $C A_{1}$ has $v_{0}, v_{1}, \cdots$ for individual variables but we use $x_{0}, \cdots, y_{0}, \cdots$, etc. to represent arbitrary variables; $\bumpeq$ is used for the formal equality; for the sake of simplicity we write $+, \cdot,-$, and $C$ for the formal operation symbols corresponding to the concrete operations; 0 and 1 are taken as abbreviations for $v_{0} \cdot-v_{0}$ and $v_{0}+-v_{0}$

Received by the editors October 1, 1970.

AMS 1969 subject classifications. Primary 0248.

Key words and phrases. Monadic algebras, equations, identities, equational classes, minimum number of variables, preservation of identities under extensions.

1 This paper has been written while the author was visiting the University of Colorado under a NATO Fellowship. 
respectively. The set of axioms characterizing $C A_{1}$ is denoted by $\Delta_{1}$ and generally, when we write " $F$ and $G$ are equivalent," or " $\tau$ is equal to $\sigma$,"or " $F \rightarrow G$," or "Mod $\mathscr{F}$ " (the class of all models of $\mathscr{F}$ ), etc. we mean these notions relative to $\Delta_{1}$, i.e., $\Delta_{1} \vdash F \leftrightarrow G, \Delta_{1} \vdash \tau \bumpeq \sigma, \Delta_{1} \vdash F \rightarrow G$, $\operatorname{Mod}\left(\mathcal{F} \cup \Delta_{1}\right)$, etc. Other usual conventions will also be freely used.

For any term $\tau$ of $L_{1},(\zeta \tau)$ represents $\tau$ if $\zeta=1$ and $-\tau$ if $\zeta=-1$; if $\zeta=0,(\zeta C \tau)$ will simply denote $\tau$.

A boolean monomial in the variables $x_{0}, \cdots, x_{n-1}(n>0)$ is a term

$$
\left(\zeta_{0} x_{0}\right) \cdot\left(\zeta_{1} x_{1}\right) \cdot \cdots \cdot\left(\zeta_{n-1} x_{n-1}\right)
$$

where $\zeta \in{ }^{n}\{1,-1\}$. The sign of $x_{j}$ in (1) is $\zeta_{j}$. We will assume that some numbering of the set of all boolean monomials in $x_{0}, \cdots, x_{n-1}$ has been fixed so that its members are $\beta_{0}, \cdots, \beta_{n^{*}-1}$ (with $\left.n^{*}=2^{n}\right)$.

$A$ monadic monomial in the variables $x_{0}, \cdots, x_{n-1}(n>0)$ is a term

$$
\left(\varepsilon_{0} C \beta_{0}\right) \cdot\left(\varepsilon_{1} C \beta_{1}\right) \cdots \cdot\left(\varepsilon_{n^{*-1}} C \beta_{n^{*-1}}\right)
$$

with $\beta_{i}, i<n^{*}$, the boolean monomials in $x_{0}, \cdots, x_{n-1}$ and $\varepsilon \in$ $n^{*}\{1,0,-1\}$. (2) will be said to be closed if $\varepsilon \in{ }^{n *}\{1,-1\}$.

An equation in $x_{0}, \cdots, x_{n-1}(n>0)$ is a formula $\tau \bumpeq \varphi$, where $\tau$ and $\varphi$ are terms and $x_{0}, \cdots, x_{n-1}$ are the variables appearing in $\tau$ and $\varphi$. An identity with bound variables $x_{0}, \cdots, x_{n-1}$ is the universal closure of such an equation. Standard equations in $x_{0}, \cdots, x_{n-1}(n>0)$ and standard identities with bound variables $x_{0}, \cdots, x_{n-1}(n>0)$ will refer to formulas $\tau \bumpeq x_{i} \cdot-x_{i}(i<n)$, with $\tau$ a closed monadic monomial in $x_{0}, \cdots, x_{n-1}$ and to the universal closure of such a formula respectively. (We use $x_{i} \cdot-x_{i}$ instead of 0 to avoid the inessential distinction: $v_{0}$ occurs in $\tau, v_{0}$ does not occur in $\tau$.)

THEOREM 1.1. Every term with variables occurring among $x_{0}, \cdots, x_{n-1}$ $(n>0)$ is equal to a finite sum of monadic monomials in $x_{0}, \cdots, x_{n-1}$.

Proof. By induction.

THEOREM 1.2. Every equation with variables occurring among $x_{0}, \cdots$, $x_{n-1}(n>0)$ is equivalent to a finite conjunction of standard equations in $x_{0}, \cdots, x_{n-1}$.

Proof. Essentially use Theorem 1.1, the normality and quasimultiplicativity of $C$.

THEOREM 1.3. Every identity is equivalent to a finite conjunction of standard identities having no more bound variables than it.

Proof. This is an immediate consequence of Theorem 1.2. 
2. Standard identities. Theorem 1.3 reduces the study of identities to the study of standard identities. In this paragraph we establish two fundamental theorems concerning them.

Let $F$ be a standard identity:

$$
F=\forall x_{0} \cdots \forall x_{n-1}\left(\mu \bumpeq x_{i} \cdot-x_{i}\right) \quad(i<n)
$$

with $\mu=\left(\varepsilon_{0} C \beta_{0}\right) \cdots\left(\varepsilon_{n^{*-1}} C \beta_{n^{*-1}}\right)$, a closed monadic monomial in $x_{0}, \cdots, x_{n-1}$. With $F$ we associate a positive integer, $\operatorname{Sgn} F$, the signature of $F$, defined by $\operatorname{Sgn} F=\operatorname{Card}(K)$ where $K=\left\{i<n^{*} \mid \varepsilon_{i}=1\right\}$.

THEOREM 2.1. Let $F$ be a standard identity.

(1) If $\operatorname{Sgn} F=0$, then $F \leftrightarrow 0 \bumpeq 0$.

(2) If $\operatorname{Sgn} F=p>0$, then $F \leftrightarrow G$, for

$$
G=\forall y_{0} \cdots \forall y_{p-1}\left(\bigwedge_{i<j<p} y_{i} \cdot y_{j} \bumpeq 0 \rightarrow C y_{0} \cdots C y_{p-1} \bumpeq 0\right) .
$$

Proof of CASE (1). In this case, $\mu=-C \beta_{0} \cdots-C \beta_{n^{*-1}}$, hence

$$
\mu \bumpeq-C\left(\sum_{i<n^{*}} \beta_{i}\right) \bumpeq-C 1 \bumpeq 0 .
$$

Proof OF CASE (2). We first prove " $G \rightarrow F$." Let $\mathfrak{A}=\langle A,+, \cdot,-, C\rangle$ be a monadic algebra model of $G$, and let $\bar{x}_{0}, \cdots, \bar{x}_{n-1}$ be arbitrary elements of $A$. Consider a bijective function $f \in{ }^{p} K$ (this is possible since Card $K=p$ ) and define for $i<p, \bar{y}_{i}=\bar{\beta}_{f(i)}$, where $\bar{\beta}_{j}, j<n^{*}$, is the element of $A$ assigned to $\beta_{j}$ under the valuation $x_{k} \rightarrow \bar{x}_{k}(k<n)$. Clearly the $\bar{y}_{i}$ are disjoint. Since $\mathfrak{A}$ is a model of $G$, we have:

hence also

$$
C \bar{y}_{0} \cdots C \bar{y}_{p-1}=0, \quad C \bar{\beta}_{f(0)} \cdots C \bar{\beta}_{f(p-1)}=0 ;
$$

$$
\left(\varepsilon_{0} C \bar{\beta}_{0}\right) \cdots\left(\varepsilon_{n^{*-1}} C \bar{\beta}_{n^{*-1}}\right)=0,
$$

by the definition of $f$ and usual boolean properties. This proves that $\mathfrak{A}$ is also a model of $F$.

To prove " $F \rightarrow G$," observe that $H \rightarrow G$ where

$$
H=\forall z_{0} \cdots \forall z_{p-1}\left(\bigwedge_{i<j<p} z_{i} \cdot z_{j} \bumpeq 0 \wedge \sum_{i<p} z_{i} \bumpeq 1 \rightarrow C z_{0} \cdots C z_{p-1} \bumpeq 0\right) .
$$

Indeed, let $\mathfrak{A}$ be a monadic algebra model of $H$ and assume $\bar{y}_{0}, \cdots, \bar{y}_{p-1}$ are disjoint elements of $A$. Let $\bar{z}_{i}=\bar{y}_{i}$ for $0<i<p$ and $\bar{z}_{0}=-\sum_{0<i<p} \bar{y}_{i}$. Clearly the $\bar{z}_{i}$ satisfy the hypothesis of $H$, hence $C \bar{z}_{0} \cdots C \bar{z}_{p-1}=0$. But, $C \bar{y}_{i} \leqq C \bar{z}_{i}$ for $i<p$, hence $C \bar{y}_{0} \cdots C \bar{y}_{p-1}=0$, which proves that $\mathfrak{A}$ is also a model of $G$.

Consequently, it suffices to show that $F \rightarrow H$. Let $\mathfrak{A}$ be a monadic algebra model of $F$; let also $\bar{z}_{0}, \cdots, \bar{z}_{p-1}$ be disjoint elements of $A$ the 
sum of which is 1 . Consider a bijective function $f \in{ }^{p} K$ and define for $j<n^{*}$,

$$
\bar{u}_{j}=\bar{z}_{i} \quad \text { for } j=f(i), \quad \bar{u}_{j}=0 \quad \text { for } j \notin K .
$$

Let, for every $i<n, I_{i}=\left\{k<n^{*} \mid \zeta_{i}^{k}=1\right\}$ and $\bar{x}_{i}=\sum_{k \in I_{i}} \bar{u}_{k}$. (Here $\zeta_{i}^{k}$ is the sign of $x_{i}$ in $\beta_{k}$.)

Since the $\bar{u}_{j}$ are disjoint and their sum is 1 ,

for any $i, j<n$.

$$
\bar{x}_{i} \cdot \bar{x}_{j}=\sum_{k \in I_{i} \cap \cap_{j}} \bar{u}_{k} \text { and }-\bar{x}_{i}=\sum_{k \in n^{*} \sim I_{i}} \bar{u}_{k},
$$

These properties show that, for $j<n$,

$$
\bar{\beta}_{j}=\left(\zeta_{0}^{j} \bar{x}_{0}\right) \cdots\left(\zeta_{n-1}^{j} \bar{x}_{n-1}\right)=\sum_{k \in J} \bar{u}_{k}
$$

where $J_{j}=\bigcap_{l<n}\left(\zeta_{l}^{j} I_{l}\right)$. (By an obvious extension of a previous notation, $\left(\zeta I_{l}\right)$ denotes $I_{l}$ if $\zeta=1$ and $n^{*} \sim I_{l}$ if $\zeta=-1$.)

But since $J_{j}=\{j\}$, it follows that $\bar{\beta}_{j}=\bar{u}_{j}(j<n)$. This result and the fact that $\mathfrak{U}$ is a model of $F$ show that

$$
\left(\varepsilon_{0} C \bar{u}_{0}\right) \cdots\left(\varepsilon_{n^{*-1}} C \bar{u}_{n^{*-1}}\right)=0
$$

from which $C \bar{z}_{0} \cdots C \bar{z}_{p-1}=0$ follows by the normality of $C$ and elementary boolean properties. This proves that $\mathfrak{U}$ is also a model of $H$ and that $F \rightarrow H$.

THEOREM 2.2. Let $F$ and $G$ be standard identities. $F \rightarrow G$ iff Sgn $G=0$ or $0<\operatorname{Sgn} F \leqq \operatorname{Sgn} G$.

Proof. If Sgn $G=0$, then $F \rightarrow G$ for any $F$ by Theorem 2.1 (1). If $0<\operatorname{Sgn} F \leqq \operatorname{Sgn} G$, Theorem 2.1 (2) clearly shows that $F \rightarrow G$. Conversely, suppose $0<\operatorname{Sgn} G<\operatorname{Sgn} F$; it is easily seen that any finite simple monadic algebra with $\operatorname{Sgn} F-1$ atoms is a model of $F$ but not of $G$. Consequently $F \rightarrow G$.

3. Some properties of identities and equational classes. In this paragraph we draw some consequences of the theorems of \$2. For each $p<\omega$, choose $F_{p}$ to be a standard identity with signature $p$ and have the least possible number of variables among such formulas.

THEOREM 3.1. If $\mathcal{F}$ is a set of identities, there exists a unique $p<\omega$ such that $\operatorname{Mod}(\mathcal{F})=\operatorname{Mod}\left(\left\{F_{p}\right\}\right)$ and there exists $F \in \mathcal{F}$ such that

(1) $F \leftrightarrow F_{p}$,

(2) $F_{p}$ has no more bound variables than $F$.

Proof. Let $\mathcal{F}^{*}$ be the set of standard identities corresponding to the identities of $\mathscr{F}$ by Theorem 1.3 and consider $\left\{\operatorname{Sgn} F^{*} \mid F^{*} \in \mathscr{F}^{*}\right.$ and Sgn $\left.F^{*}>0\right\}$. If this set is empty, we let $p=0$ and if it is not, we let $p$ be 
its smallest element. In both cases we take for $F$ an element of $\mathcal{F}$ such that one of the standard identities, say $F^{\prime}$, corresponding to $F$ by 1.3 has signature $p$. By the definition of $F_{p}$ and the choice of $F^{\prime}, F_{p}$ has no more bound variables than $F^{\prime}$; and by the choice of $F, F^{\prime}$ has no more bound variables than $F$; this proves (2). The remaining statements of the theorem are reduced by 1.3 and 2.2 to trivial comparisons of signatures of standard identities.

COROLlary 3.2. Every identity is equivalent to a standard identity.

In order to interpret Theorem 3.1 in terms of equational classes, we let $H_{p}=\operatorname{Mod}\left(\left\{F_{p+1}\right\}\right)$ for $p<\omega$ and $H_{\omega}=\operatorname{Mod}\left(\left\{F_{0}\right\}\right)$.

THEOREM 3.3 (MONK). (1) The lattice of equational classes of monadic algebras is the chain: $H_{0} \subset H_{1} \subset \cdots \subset H_{\omega}$.

(2) For every $p \leqq \omega, H_{p}$ is finitely axiomatizable.

(3) For every $p \leqq \omega$, the equational theory of $H_{p}$ is decidable.

Proof. For (1), apply 3.1 and 2.2 ; (2) is trivial. To prove (3), remark that the proofs of $1.1,1.2,1.3$, and 3.1 together with Theorem 2.2 implicitly describe a decision method.

This theorem is essentially due to Monk (see [2]); it is readily seen that $H_{\omega}=C A_{1}$ and that for $p<\omega$, our $H_{p}$ coincide with Monk's $K_{p}$ ( $K_{p}$ is the equational closure of the class of simple finite monadic algebras with $p$ atoms).

THEOREM 3.4. For $0<p<\omega$, the minimum number of bound variables needed in an identity characterizing $H_{p}$ (relative to $\Delta_{1}$ ) is the smallest $n$ such that $2^{n} \geqq p+1$. For $H_{0}$ and $H_{\omega}$, this number is 1 .

Proof. For $H_{\omega}$, take $\forall v_{0}(0 \bumpeq 0)$ and for $H_{0}$, take $\left(\forall v_{0}\right)(0 \bumpeq 1)$. For $0<p<\omega$, let $n_{0}$ denote the smallest $n$ such that $2^{n} \geqq p+1$. The definitions of standard identity, of signature and of $F_{p+1}$ show that $F_{p+1}$ has exactly $n_{0}$ bound variables. On the other hand, if $F$ is an identity characterizing $H_{p}$, Theorem 3.1 applied to $\mathcal{F}=\{F\}$ shows that $F$ has at least as many variables as $F_{p+1}$ i.e., at least $n_{0}$ variables.

We now turn to the proof that identities are preserved under certain types of extension:

THEOREM 3.5. Let $\mathfrak{A}=\langle A,+, \cdot,-, C\rangle$ be a monadic algebra and $\mathfrak{U}^{\prime}=\left\langle A^{\prime},+, \cdot,-, C^{\prime}\right\rangle$ be a monadic algebra extending $\mathfrak{U}$ and satisfying the following condition:

(P) There exists $B \subseteq A^{\prime}$ such that

(1) for every $a \in A^{\prime}$,

$$
C^{\prime} a=\sum_{a \geqq b \in B} \prod_{b \leqq c \in A} C c,
$$


(2) (separation) for every $b, b^{\prime} \in B, b \cdot b^{\prime}=0$, there exist $d, d^{\prime} \in A$ such that

$$
b \leqq d, \quad b^{\prime} \leqq d^{\prime} \quad \text { and } d \cdot d^{\prime}=0 .
$$

If $\mathfrak{A}$ is a model of an identity $F$, then $\mathfrak{U}^{\prime}$ is also a model of $F$.

Proof. By 3.2, it suffices to show this for a standard $F$. If $\operatorname{Sgn} F=0$, apply Theorem 2.1 (1). Suppose now that $\operatorname{Sgn} F=p>0$. By part (1) of condition (P) and 2.1 (2) it suffices to show that if $b_{0}, \cdots, b_{p-1}$ are disjoint elements of $B$, then

$$
\prod_{b_{0} \leqq c_{0} \in A} \cdots \prod_{b_{\nu-1} \leqq c_{p-1} \in A} C c_{0} \cdots C c_{p-1}=0 .
$$

The separation property (2) shows that we can choose disjoint $d_{0}, \cdots$, $d_{p-1} \in A$ such that $d_{i} \geqq b_{i}, i<p$. Consequently, the left member of (3) is smaller than or equal to

$$
C d_{0} \cdots C d_{p-1}
$$

and this element is 0 since $\mathfrak{A}$ is a model of $F$ (apply again 2.1 (2)).

For the notions of canonical embedding algebra and completion algebra, we refer to $[1, \S 2.7]$ and [3] respectively.

COROLlaRY 3.6. Let $\mathfrak{A}$ be a monadic algebra. If $\mathfrak{A}$ is a model of an identity $F$, then the canonical embedding algebra of $\mathfrak{A}$ and the completion algebra of $\mathfrak{A}$ are also models of $F$.

Proof. Condition (P) of Theorem 3.5 is satisfied if we choose $B$ as the set of all atoms of the canonical embedding algebra in the first case, and $B=A$ in the second case.

\section{BIBLIOGRAPHY}

1. L. Henkin, J. D. Monk and A. Tarski, Cylindric algebras. I, North-Holland, Amsterdam (to appear).

2. J. D. Monk, On equational classes of algebraic versions of logic. I, Math. Scand. 27 (1970), 53-71.

3. - Completions of boolean algebras with operators, Math. Nachr. (to appear).

Department of Mathematics, University of Montreal, Montreal, Quebec, Canada

Department of Mathematics, University of Louvain, Louvain, Belgium

Current address: Institut Mathématique, Celestijnenlaan, 200D, 3030 Heverlee, Belgium. 